\title{
Rate Between Examination of EGFR Mutation Blood Plasma Sample (ctDNA) With Cytological/Histopathological Sample in Adenocarcinoma Lung Cancer
}

\author{
Dian Utami Wulaningrum*, Ana Rima Setijadi, Suradi \\ ${ }^{1}$ Department of Pulmonology and Respiratory Medicine Faculty of Medicine, \\ Universitas Sebelas Maret, dr. Moewardi Hospital, Surakarta
}

\begin{abstract}
Background: Most adenocarcinoma lung cancer which is found at an advanced stage with cytology / histopathological samples is hardly available. Examinations EGFR mutations as a biomarker for adenocarcinoma lung cancer using cytological/histopathological sample (tissue biopsy or resection and cytologi) and ctDNA blood plasma. Examination of EGFR mutations in ctDNA blood plasma sampling is simpler and easier, which also can be used as predictive and prognostic markers in non-small cell carcinoma lung cancer patients. The purpose of this study is to determine and analyse the degree of compatibility between examination of EGFR mutations by blood plasma (ctDNA) samples with the examination of cytology/histopathological EGFR mutations in adenocarcinoma lung cancer.
\end{abstract}

Methods: Diagnostic test research, by taking medical records of patients with adenocarcinoma lung cancer from January to September 2019 at Dr. Moewardi Surakarta, who was examined by EGFR mutations in cytology / histopathology and ctDNA samples.

Result: The Subjects of this study were 73 patients with adenocarcinoma lung cancer. The level of compatibility of ctDNA with EGFR mutations in cytology/histopathology samples was categorized as moderate and statistically significant (Kappa=0.459; $\mathrm{P}=0.0001$ ), with a sensitivity value of $54.5 \%$ and a specificity of $90 \%$.

Conclusion: The high/moderate concordance between the different DNA sources for egfr mutations (cytology/histopathology) and ctDNA.

Keywords: lung cancer, adenocarcinoma, EGFR, ctDNA

*Correspondence: Dian Utami Wulaningrum - dianutamiw@gmail.com

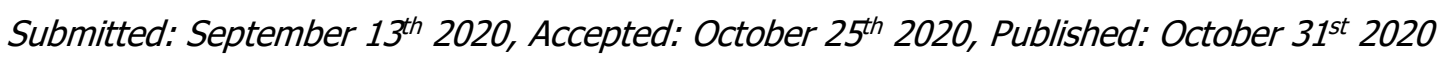




\section{INTRODUCTION}

Non-small cell carcinoma lung cancer is the leading cause of cancerrelated death. Lung cancer cases are found at an advanced stage due to non-specific symptoms (57\% of lung cancer cases in the US are detected by metastasis). Pulmonary adenocarcinoma represents about $50 \%$ of lung cancers and $60 \%$ of LCMC. EGFR examination becomes a routine test after the diagnosis of pulmonary adenocarcinoma has been established for the selection of patients receiving tyrosine kinase inhibitors (TKIs). EGFR mutation examination samples derived from tissue biopsy/resection and cytology are often not available because tumor biopsy is an invasive and high-risk procedure. Liquid biopsy can detect biomarkers associated with tumors to diagnose lung cancer earlier and more safely. ${ }^{1-7}$

The ASSESS study in the subset of Spanish patients, the good concordance (almost 90\%) between the different DNA sources supports the use of plasma samples when tumor tissue is not available. Zhang et al's study about ctDNA assessment of EGFR mutation status in Chinese patients with advanced non-small cell lung cancer in real-world setting conclude ctDNA based EGFR mutation test is feasible and could be a surrogate when tissue biopsy is not available. ${ }^{1-7}$

The purpose of this study was to determine and analyze the degree of concordance of the EGFR mutations examination originating from blood plasma samples (ctDNA) with examinations derived from cytology /histopathology. This study hope to be able to provide scientific information regarding the concordance of EGFR mutations examination originating from blood plasma (ctDNA) with cytology/histopathology samples and as basic data for further research.

\section{METHOD}

This research is a diagnostic test research. The study population was all lung cancer populations with adenocarcinoma types, naïve treatment. The diagnosis was confirmed by the presence of lung mass images on CT-scans and cytology/histopathology examinations were performed for both primary tumours and metastatic lesions and EGFR mutations were examined from cytology/histopathology samples and blood plasma (ctDNA) who underwent outpatient and inpatient care at $\mathrm{Dr}$. Moewardi Surakarta from JanuarySeptember 2019.

The inclusion criteria is patients with adenocarcinoma lung cancer, the diagnosis is confirmed by the presence 
of lung mass images on CT-scan and cytology/histopathological examination of both primary tumors and metastatic lesions with adenocarcinoma results, which are subjected to ctDNA examination and examination of EGFR mutation cytology/histopathology. The exclusion criteria are the patient's medical records are missing or incomplete, examination of EGFR mutations is performed in only one of the two tests for EGFR mutations (examination of EGFR mutations from cytology/histopathology only, or blood plasma ctDNA only). The sample of this study were obtained from the medical record data of adenocarcinoma lung cancer patients at Dr. Moewardi Hospital.

Examination of EGFR mutations with cytological samples (pleural effusion, bronchus rinse, bronchial swab, FNAB) or histopathology fixed with a neutral buffer of $10 \%$ formalin on cell blocks or slides stained / not stained with tumor cell counts greater than 200 cells and containing more From $50 \%$ tumor cells, both primary and metastatic lesions using the DNA extraction method with the Qiagen QIAamp® DNA Micro kit, mutation analysis using PCR HRM, fragment analysis, direct sequencing and amoyDx. $\quad 100 \%$ specificity. ctDNA examination using Therascreen EGFR
Plasma RGQ PCR Kit.

Sample criteria were plasma EDTA from adenocarcinoma positive patients. Minimum plasma volume of $4.5 \mathrm{ml}$. One job can be done up to a maximum of 16 samples or 1 kit can be divided by $3 x$ runs with samples per one run. Plasma sample separation and storage procedures prior to EGFR examination was fresh blood samples should be centrifuged immediately to separate the plasma within 2 hours if stored at room temperature or 8 hours if stored at $4^{\circ} \mathrm{C}$. Fresh blood centrifuge at $2000 \mathrm{xg}$ for 10 minutes. Separate the plasma into a new tube. The separated plasma can be stored at $-20^{\circ} \mathrm{C}$ for 4 weeks or $-80^{\circ} \mathrm{C}$ for longer storage. Avoid re-freezing. Only thaw if an examination is to be performed. Thaw the plasma at room temperature on the day of the EGFR examination. Centrifuge a second time at a speed of $16,000 \times \mathrm{g}$ for 10 minutes at $4^{\circ} \mathrm{C}$ (at fixed angle rotor). Separate the clear portion of the plasma into a $14 \mathrm{ml}$ sample tube $(14 \mathrm{ml}$ Falcon $\AA$ polystyrene $17 \times 100 \mathrm{~mm}$ round bottom tube (BD, paint no. 352051)) to continue the extraction process. Ethical clearance approved by The Health Research Ethics Committee Dr. Moewardi General Hospital.

Data analysis was performed using SPSS 21 for Windows. In this 
study, the analysis is presented with a frequency distribution and percentage. The degree of concordance between the results of the EGFR mutation examination with blood plasma samples (ctDNA) and cytology/histopathology samples was calculated by means of a test of agreement (Kappa Cohen) with kappa values:> 0.8 (very good suitability), kappa: 0.6 - 0.8 (good suitability), kappa: 0.4 - 0.6 (moderate suitability) and kappa: $<0.4$ (less suitability). The degree of concordance between ctDNA examination results and EGFR mutations (cytology/ histopathological samples) is presented in the $2 \times 2$ table. We calculate sensitivity, specificity, positive and negative predictive value.

\section{RESULT}

The research was conducted in the medical record room of Dr. Moewardi Surakarta from October 2019 to November 2019. The results of recording the medical records of patients with adenocarcinoma lung cancer that were carried out by EGFR examination were obtained from cytology/histopathology and blood plasma (ctDNA) samples from both primary tumors and metastatic lesions obtained by 73 people. The scheme sample collection is on Figure 1.

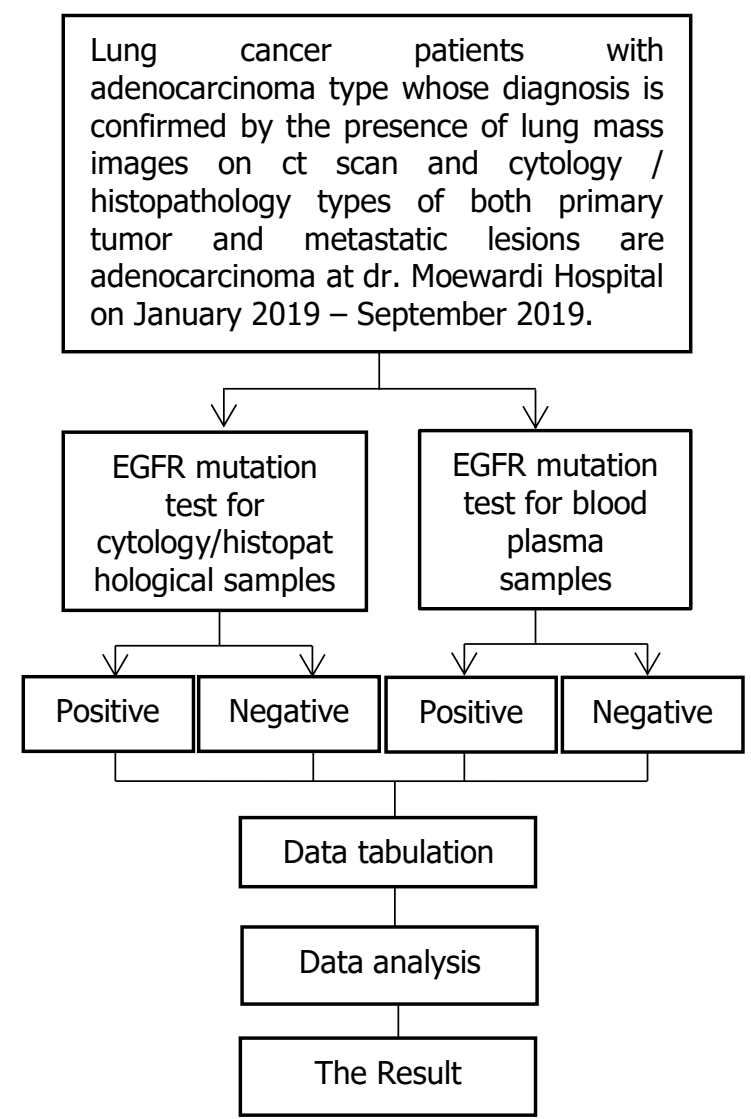

Figure 1 . The scheme of sample collection

Lung cancer patients with adenocarcinoma types based on the table above were 35 men (47.9\%) and 38 women $(52.1 \%)$. The majority of patients with adenocarcinoma lung cancer in the study were $\geq 40$ years by 67 people $(91.8 \%)$, while the rest $<40$ years were 6 people (8.2\%). 
Table. 1 Basic characteristics of research subjects

\begin{tabular}{|c|c|c|}
\hline Characteristics & Frequency & Percentage \\
\hline \multicolumn{3}{|l|}{ Gender } \\
\hline Male & 35 & 47.9 \\
\hline Women & 38 & 52.1 \\
\hline \multicolumn{3}{|l|}{ Age } \\
\hline$<40$ years & 6 & 8.2 \\
\hline$\geq 40$ years & 67 & 91.8 \\
\hline \multicolumn{3}{|l|}{ EGFR Mutation Results } \\
\hline No mutations & 39 & 53.4 \\
\hline Del Exon 19 & 18 & 24.7 \\
\hline Exon $21 \mathrm{~L} 861 \mathrm{Q}$ & 1 & 1.4 \\
\hline Exon 21 L858R & 10 & 13.7 \\
\hline Exon $20 \mathrm{~T} 790 \mathrm{M}+$ Exon 21 L858R & 1 & 1.4 \\
\hline Del Exon 19 + Exon 21 L858R & 2 & 2.7 \\
\hline Exon $21 \mathrm{~L} 858 \mathrm{R}+$ Exon $21 \mathrm{~L} 861 \mathrm{Q}$ & 2 & 2.7 \\
\hline \multicolumn{3}{|l|}{ ctDNA results } \\
\hline No mutations & 48 & 65.8 \\
\hline Del Exon 19 & 16 & 21.9 \\
\hline Exon 21 L858R & 7 & 9.6 \\
\hline Exon $20 \mathrm{~T} 790 \mathrm{M}+$ Exon 21 L858R & 2 & 2.7 \\
\hline \multicolumn{3}{|l|}{ Smoking History } \\
\hline Yes & 30 & 41.1 \\
\hline Not & 43 & 58.9 \\
\hline \multicolumn{3}{|l|}{ Stadium } \\
\hline IIIC & 4 & 5.5 \\
\hline IV & 69 & 94.5 \\
\hline \multicolumn{3}{|l|}{ Metastasis } \\
\hline There is no & 4 & 5.5 \\
\hline Pleural effusion & 25 & 34.2 \\
\hline Bone metastasis & 6 & 8.2 \\
\hline Metastatic liver & 3 & 4,1 \\
\hline Brain metastasis & 2 & 2.7 \\
\hline Pneumonic type & 3 & 4,1 \\
\hline Pleural effusion, bone, liver metastases & 5 & 6.8 \\
\hline Pleural effusion, bone metastasis & 8 & 11 \\
\hline Pleural effusion, bone, brain metastasis & 1 & 1.4 \\
\hline Pleural effusion, colli lymphadenopathy & 2 & 2.7 \\
\hline Bone, brain metastasis, pneumonic type & 2 & 2.7 \\
\hline Bone metastases, colli lymphadenopathy & 1 & 1.4 \\
\hline Bone metastasis, pneumonic type & 2 & 2.7 \\
\hline Pleural effusion, liver metastases & 5 & 6.8 \\
\hline
\end{tabular}




\begin{tabular}{lcc}
\hline \multicolumn{1}{c}{ Characteristics } & Frequency & Percentage \\
\hline Bone, liver metastases & 3 & 4,1 \\
Bone, brain metastasis & 1 & 1.4 \\
Cytology / Histopathology Samples & & \\
TTNA & 40 & 54.8 \\
Bronchial brushing & 7 & 9.6 \\
Bronchial washing/BAL & 4 & 5.5 \\
Endobronchial biopsy & 6 & 8.2 \\
FNAB & 7 & 9.6 \\
Thoracocentesis & 8 & 11.0 \\
Core Needle Biopsy & 1 & 1.4 \\
\hline
\end{tabular}

The results of ctDNA examination were obtained as follows, there were no mutations in 48 people $(65.8 \%), 16$ people with exon 19 deletions (21.9\%), 7 people with exon 21 L858R (9.6\%), 20 T790M exon. + Exon 21 L858R there are 2 people (2.7\%). Smoking history was also found on the patients there were 30 people $(41.1 \%)$ smoked while 43 people (58.9\%) did not smoke. The stage in the subjects of this study was an advanced stage, there were 4 people (5.5\%) with stage IIIC, while in stage IV there were 69 people (94.5\%).

The table above also shows that 4 patients $(5.5 \%)$ had no metastases, while the other 69 patients had metastases (94.5\%). Pleural effusion occurred in 25 people (34.2\%), this is the most common metastasis in the subjects of this study. Table 1 shows that the metastases that occurred in the subjects of this study did not only occur in one place but there were also several places such as pleural effusion, bone metastasis and brain metastasis simultaneously.

Table 2. Results of EGFR mutations in cytological/histopathological samples by sex

\begin{tabular}{lccc}
\hline \multirow{2}{*}{ Sex } & \multicolumn{2}{c}{ EGFR Mutation Results } & \multirow{2}{*}{ Total } \\
\cline { 2 - 3 } & Negative & Positive & \\
\hline Male & $23(31.5 \%)$ & $12(16.4 \%)$ & $35(47.9 \%)$ \\
Women & $17(23.3 \%)$ & $21(28.8 \%)$ & $38(52.1 \%)$ \\
Total & $40(54.8 \%)$ & $33(45.2 \%)$ & $73(100 \%)$ \\
\hline
\end{tabular}

The result of EGFR mutations based on cytology/histopathological samples based on sex was more prevalent in 21 women (28.8\%) compared to men, namely 12 (16.4\%) shown in Table 2.

Table 3. Results of ctDNA by sex

\begin{tabular}{lccc}
\hline \multirow{2}{*}{ Sex } & \multicolumn{2}{c}{ ctDNA results } & \multirow{2}{*}{ Total } \\
\cline { 2 - 3 } & Negative & Positive & \\
\hline Male & $27(37.0 \%)$ & $8(10.9 \%)$ & $35(47.9 \%)$ \\
Women & $21(28.8 \%)$ & $17(23.3 \%)$ & $38(52.1 \%)$ \\
Total & $48(65.8 \%)$ & $25(34.2 \%)$ & $73(100 \%)$ \\
\hline
\end{tabular}


Table 4. Kappa test results between ctDNA and EGFR mutation examination of cytology / histopathology samples

\begin{tabular}{cccccc}
\hline \multirow{2}{*}{ CTDNA } & \multicolumn{2}{c}{ EGFR mutations } & Total & Kappa test & P \\
\cline { 2 - 3 } & Positive & Negative & & & \\
\hline Positive & 18 & 4 & 22 & 0.459 & 0.0001 \\
Negative & 15 & 36 & 51 & & \\
Total & 33 & 40 & 73 & & \\
\hline
\end{tabular}

Table 5. Sensitivity and specificity tests

\begin{tabular}{lccc}
\hline \multirow{2}{*}{ ctDNA } & \multicolumn{2}{c}{ EGFR mutations } & \multirow{2}{*}{ Total } \\
\cline { 2 - 3 } & Positive & Negative & \\
\hline Positive & 18 & 4 & 22 \\
Negative & 15 & 36 & 51 \\
$\quad$ Total & 33 & 40 & 73 \\
\hline Sensitivity & $: 0.545$ & Specificity & $: 0.900$ \\
PPR & $: 0.818$ & NEV & $: 0.706$ \\
PLR & $: 5,450$ & NLR & $: 0.505$
\end{tabular}

Note: PPR=Positive Probability Ratio, NEV: Negative Estimated Value, PLR: Positive Likelihood Ratio, NLR: Negative Likelihood Ratio

The results of ctDNA examination based on sex in Table 3 show that there were more mutations in 17 women (23.3\%) while in men there were 8 people (10.9\%). The ctDNA examination compared with EGFR mutations with cytology/histopathology samples is called true positive taking into account the same mutation points. The kappa test results show a value of 0.459 which means the level of concordance between the results ctDNA with EGFR examination of cytology/histopathological samples with moderate levels, $P=0.0001$ $(P<0.05)$, which means that the suitability of ctDNA with cytology/histopathology was statistically significant.

The diagnosis of EGFR mutations with ctDNA samples obtained a sensitivity of $54.5 \%$, which means that $54.5 \%$ of the diagnosis of EGFR mutations in cytology/histopathology samples with positive results could be detected by positive ctDNA examination and the specificity value of ctDNA measurements obtained in this study was $90.0 \%$ means that $90.0 \%$ of the diagnosis of negative EGFR mutations will be excluded in ctDNA positive patients.

On examination, the NDP value was $81.8 \%$, which means that if the ctDNA test was positive, then there was an $81.8 \%$ chance of a positive EGFR mutation diagnosis. While the NDN value is $70.6 \%$, which means that if the ctDNA is negative, there is a $70.6 \%$ chance of diagnosing an EGFR mutation with a negative result.

The RKP value is 5.45 , which means that the possibility of a patient with a positive ctDNA result will get a 
diagnosis of an EGFR mutation with a positive result of 5.45 times greater than a negative ctDNA. The RKN value is 0.505 , which means that the likelihood that a patient with a negative ctDNA measurement value will get a diagnosis of an EGFR mutation with a positive result is 0.505 times less than a patient with ctDNA with a positive result.

\section{DISCUSSION}

This study found a total sample of adenocarcinoma lung cancer patients who were examined for ctDNA and EGFR mutations with 73 cytology/ histopathological samples. The subjects of this study were 38 women (52.1\%) who suffered from adenocarcinoma lung cancer, this is in accordance with the histological prevalence of adenocarcinoma tumors which occurred more in women over three decades, with the incidence increasing slowly. ${ }^{8}$

The age of the patients as the subjects in this study tend to be $\geq 40$ years with $91.8 \%$, where age $\geq 40$ years was a high risk of lung cancer. ${ }^{9}$ EGFR mutations in cytology /histopathological samples in this study occurred many deletions of exon 19, there were 18 people (24.7\%) and 10 exon 21 L858R mutations (13.7\%), Pal et al stated that the most common mutations were in exon 19 and exon 21 L858R2. Lyu et al's research in 2018 showed that ctDNA examination had higher accuracy against deletions of exon 19 and exon 21 L858R, in a study conducted at dr. Moewardi showed that exon 19 deletions were 16 people (21.9\%) and exon 21 L858R, namely 7 people $(9.6 \%){ }^{10}$

This study shows that $58.9 \%$ of patients do not smoke, this may be due to the number of female subjects more than men. Table 1 shows that the subjects of this study are advanced stages patients, with stage IIIC and IV with metastases in various places, Cheng et al in 2017 stated that $57 \%$ of patients in the US were diagnosed at an advanced stage and had metastasis. Qiu et al's (2015) study cited that patients with advanced stages have high levels of circulating tumor DNA, the current hypothesis indicates that the amount of ctDNA is associated with tumor volume and metastasis. ${ }^{11}$

The results of this study indicate that the number of cytological samples (TTNA, bronchial brushing, bronchial rinse, $F N A B$, pleural fluid) was higher than the histopathological samples (core biopsy, forceps biopsy). A study conducted by Yatabe in 2015 showed that $98 \%$ of the data for examining EGFR mutations in Indonesia were 
cytology samples and 2\% biopsy samples. ${ }^{12}$

Hlinkova's research in 2013 showed that $85.9 \%$ of cytological samples were used to examine EGFR mutations. Two-thirds of patients are at an advanced stage when they were diagnosed for lung cancer, only small biopsy or cytology specimens are available for EGFR examination in the majority of patients. Any type of small biopsy or cytological specimen is suitable for examination of mutations that have been confirmed by reports from various laboratories around the world. ${ }^{12}$

The results of EGFR mutations in cytology/histopathology samples (table 2) and the results of EGFR mutations in blood plasma samples (ctDNA) in table 3 based on sex showed that more women were 22 subjects and 17 subjects were male. These results are in accordance with the study by Zhang et al. In 2016 which concluded that mutations were more common in women $(43.7 \%)$ than in men (24\%). The clinic pathological features that correlate with the EGFR mutation include East Asian ethnicity, adenocarcinoma histology, women and a history of never smoking. ${ }^{13}$

The kappa test results in table 4 show a value of 0.459 , which means the level of concordance between the
ctDNA results and the examination of the EGFR mutation in the cytology/ histopathology sample is moderate, with $\mathrm{P}=0.0001$ which means statistically significant with a sensitivity of $54.5 \%$, specificity of $90 \%$, PPR 81 , $8 \%$, NEV $70.6 \%$ (table 5). These results differ from the 2016 ASSESS study regarding the suitability level of $90 \%$, $50 \%$ sensitivity, $80 \%$ PPR, and $91 \%$ NEV, while the 2014 IFUM study had a $95 \%$ conformance rate, $73 \%$ sensitivity, 99\% specificity, 94\% PPR, and $95 \%$ NEV. ${ }^{14}$ The IGNITE Study in 2013 - 2014, mutation status concordance between 2581 matched tissue/cytology and plasma samples: $80.5 \%$ (sensitivity $46.9 \%$, specificity $95.6 \%) .{ }^{15}$

Zhang et al's study about ctDNA assessment of EGFR mutation status in Chinese patients with advanced nonsmall cell lung cancer in real-world setting, in this study 35 patients had both tissue and plasma samples and the detection concordance was $68.6 \%$ (24/35). ${ }^{3}$ Mao et al in 2013 presents the meta-analysis of diagnostic tests for EGFR mutation in blood using EGFR mutation in tumor tissues as the gold standard, the sensitivity, specificity, and concordance rate were 0.61 (95\% CI $0.50-0.71), 0.90$ (95\% CI $0.85-$ 0.94 ), and 0.79 (95\% CI $0.73-0.84$ ), respectively. ${ }^{15}$ 
This difference in the level of concordance may be due to the fact that the specimens examined are not optimal, this can occur from blood collection, specimen treatment, delivery of specimens from the collection location to the examination site, DNA purification, and gaps from knowledge. 11,14

The results of this study indicate that ctDNA has high specifications and is an effective biomarker for detection of EGFR mutation status. These results are consistent with research conducted by Qiu et al. 2015 with the conclusion that ctDNA is an effective method for detecting EGFR mutation status in KPKBSK, based on high diagnostic accuracy and specificity, ctDNA can be the main screening test for NSCLC and ctDNA analysis methods. Standardized and validation still needs to be developed. ${ }^{11}$

\section{CONCLUSION}

The level of concordance between the examination of the EGFR mutation of blood plasma samples (ctDNA) and the examination of the EGFR mutation of the cytology / histopathology sample was in the moderate category (kappa test 0.459) and statistically significant, with a sensitivity value of $54.5 \%$ and a specificity of $90 \%$.

\section{REFERENCES}

1. Cheng TYD, Cramb SM, Baade PD, Youlden DR, Nwogu C, Reid ME. The international epidemiology of lung cancer: Latest trends, disparities, and tumor characteristics. Journal of Thoracic Oncology. 2016;11(10):1653-1671.

2. Arriola E, Paredes-Lario A, GarcíaGomez R, et al. Comparison of plasma ctDNA and tissue/ cytology-based techniques for the detection of EGFR mutation status in advanced NSCLC: Spanish data subset from ASSESS. Clinical and Translational Oncology. 2018;20(10):1261-1267.

3. Zhang $S$, Zhu $L$, Chen $X$, et al. CtDNA assessment of EGFR mutation status in Chinese patients with advanced non-small cell lung cancer in real-world setting. Journal of Thoracic Disease. 2018;10(7):4169-4177.

4. Pal $P, M C, M S ~ T$. Pulmonary adenocarcinoma - pathology and molecular testing. In: Horn L. Pulmonary adenocarcinoma: approaches to treatment. 1st ed. Nashville: Elsevier Inc .; 2019. p. 13-33

5. Shim HS, Chung JH, Kim L, et al. Guideline recommendations for EGFR mutation testing in lung 
cancer: Proposal of the korean cardiopulmonary pathology study group. Korean Journal of Pathology. 2013;47(2):100-106.

6. Wu Z, Yang Z, Dai Y, Zhu Q, Chen LA. Update on liquid biopsy in clinical management of non-small cell lung cancer. OncoTargets and Therapy. 2019;12:5097-5109.

7. Heydt C, Michels S, Thress KS, Bergner $S$, Wolf J, Buettner R. Novel approaches against epidermal growth factor receptor tyrosine kinase inhibitor resistance. Oncotarget. 2018;9(20):15418-15434.

8. Egleston $\mathrm{BL}$, Meireles SI, Flieder DB, Clapper ML. Population-Based Trends in Lung Cancer Incidence in Women. Seminars in Oncology. 2009;36(6):506-515.

9. Zaini J, Syahrudin E, Andarini S. Non-Small Cell Lung Cancer. In: Rasmin M, Jusuf A, Yunus $F$, Amin $M$, Syaifudin T, Nawas MA et al, editors. Textbook of Pulmonology and Respiration Medicine. 1st ed. Jakarta: University of Indonesia Publishing; 2018. p. 9-22

10. Lyu M, Zhou J, Ning K, Ying B. The diagnostic value of circulating tumor cells and ctDNA for gene mutations in lung cancer. OncoTargets and Therapy. 2019;12:2539-2552.
11. Qiu $M$, Wang J, $X u Y$, et al. Circulating Tumor DNA Is Effective for the Detection of EGFR Mutation in Non-Small Cell Lung Cancer: A Meta-analysis. 2014.

12. Tsao M, Wistuba I, Yatabe $\mathrm{Y}$. EGFR testing. doi:In: Mok TS, Carbone DP, Hirsch FR, editors. IASLC atlas of EGFR testing in lung cancer. 1st ed. North Fort Myers: Editorial Rx Press; 2017. p. 19-25

13. Zhang $\mathrm{YL}$, Yuan JQ, Wang $\mathrm{KF}$, et al. The prevalence of EGFR mutation in patients with nonsmall cell lung cancer: A systematic review and metaanalysis.

Oncotarget. 2016;7(48):78985-78993.

14. Reck M, Hagiwara $K$, Han $B$, et al. CtDNA determination of EGFR mutation status in European and Japanese patients with advanced NSCLC: The ASSESS study. Journal of Thoracic Oncology. 2016;11(10):1682-1689.

15. Han $B$, Tjulandin $S$, Hagiwara $K$, et al. EGFR mutation prevalence in Asia-Pacific and Russian patients with advanced NSCLC of adenocarcinoma and nonadenocarcinoma histology: The IGNITE study. Lung Cancer. 2017;113:37-44. 